\title{
Flexible Ceramic Nanofibrous Aerogels with Hierarchically Entangled Graphene Networks Enable Full-Frequency Noise Absorption
}

\section{Dingding Zong}

State Key Laboratory for Modification of Chemical Fibers and Polymer Materials, College of Materials Science and Engineering, Donghua University, Shanghai 201620, China.

\section{Leitao Cao}

Donghua University

\section{Xia Yin}

State Key Laboratory for Modification of Chemical Fibers and Polymer Materials, College of Textiles, Donghua University, Shanghai 201620, China.

\section{Yang Si}

Donghua University

\section{Shichao Zhang}

Innovation Center for Textile Science and Technology, Donghua University, Shanghai 200051, China.

\section{Jianyong Yu}

Donghua University

Bin Ding ( $\sim$ binding@dhu.edu.cn )

Donghua University

\section{Article}

Keywords: traffic noise pollution, noise absorption, flexible ceramic nanofibrous aerogels (GCNAs)

Posted Date: March 30th, 2021

DOI: https://doi.org/10.21203/rs.3.rs-337332/v1

License: (c) (1) This work is licensed under a Creative Commons Attribution 4.0 International License. Read Full License

Version of Record: A version of this preprint was published at Nature Communications on November 15th, 2021. See the published version at https://doi.org/10.1038/s41467-021-26890-9. 


\section{Abstract}

Traffic noise pollution has posed a huge burden to the global economy, ecological environment, and human health. However, most present traffic noise reduction materials suffer from a narrow absorbing band, high weights, and poor temperature resistance. Here, we demonstrate a facile strategy to create flexible ceramic nanofibrous aerogels (GCNAs) with hierarchically entangled graphene networks, which integrate unique hierarchical structures of opened cells, closed-cell walls, and entangled networks. Under the precondition of independent of chemical crosslinking, high enhancement in buckling and compression performances of GCNAs is achieved by forming hierarchically entangled structures in all three-dimensional space. Moreover, the flexible GCNAs show striking full-frequency noise absorption performances (noise reduction coefficient of 0.56 in $63 \sim 6300 \mathrm{~Hz}$ ) and lightweight features $\left(9.3 \mathrm{mg} \mathrm{cm}^{-3}\right)$, together with robust temperature-invariant stability in $-100 \sim 500^{\circ} \mathrm{C}$. This strategy paves the way for the design of novel fibrous materials for highly efficient full-frequency noise absorption.

\section{Introduction}

With the rapid development of the transportation industry, traffic noise pollution, as the hidden killer of the global economy, ecological environment, and human health, has become increasingly serious. ${ }^{1,2}$ According to the World Health Organization, there are more than 466 million people worldwide who suffer from disabling hearing loss that is mostly caused by exposure to noise, which causes total annual economic losses of over $\$ 750$ billion. ${ }^{3}$ Therefore, efficient traffic noise reduction methods are of great importance for eliminating unwanted sound. Fibrous materials with the advantages of porous structure and tortuous channels, which could enhance friction and dissipation of sound waves, thus showing good high-frequency absorption performance. ${ }^{4}$ Previous efforts were focused on using cost-effective fibrous materials as the core components for constructing noise-absorber. ${ }^{4-6}$ However, due to the inherent limitations of large fiber diameter (usually $>5 \mu \mathrm{m}$ ) and low porosity (typically $<60 \%$ ), the fatal defects of poor absorption of low-frequency noise that is easily produced by vehicles remain for the conventional microfibrous noise-absorbing materials. ${ }^{7-9}$ To address this problem, it is necessary to increase the thickness or density of the fibrous materials, while which in turn lead to high weights $\left(>50 \mathrm{mg} \mathrm{cm}^{-3}\right)$ and decreasing consumption of high-frequency sound waves. ${ }^{10-12}$ Moreover, the vehicle's space left for sound-absorbing materials is limited, and increasing the density will improve fuel consumption, which violates the principle of energy saving. Most importantly, the flammable characteristic of synthetic fibrous sound-absorbing materials gives rise to poor thermostability, which is dangerous especially using near the engine and hood. ${ }^{13-16}$ Therefore, great efforts are required to create a specialized and practicable fibrous sound-absorbing material for the efficient reduction of full-frequency noise.

Compared with microfibers, electrospun nanofibers show effective improvement in noise absorption performances due to their small diameter and large specific surface area. ${ }^{8,17,18}$ However, lamellar deposition character usually induces nanofibers to assemble into densely packed two-dimensional (2D) membranes (thickness $<50 \mu \mathrm{m}$ and porosity $<80 \%$ ), thus greatly limiting the dissipation of acoustic 
energy. ${ }^{19-22}$ Most recently, three-dimensional (3D) nanofibrous aerogel, as the forefront of advanced fibrous materials, combining small fiber diameter, high porosity $(>95 \%)$, and bulk structure, have shown vast perspectives in the field of traffic noise reduction. ${ }^{23-26} \mathrm{~A}$ few polymer-based nanofibrous soundabsorbing aerogels have been successfully constructed and show enhanced sound absorption performance in contrast to 2D membranes. ${ }^{19,27-29}$ Unfortunately, due to the monotonous and uncontrollable structure, totally opened cells, and highly connected macropore structure, existing nanofibrous aerogels still face a narrow absorbing band $(>1000 \mathrm{~Hz})$ and poor mechanical performance. ${ }^{19,27}$ Although the extra chemical crosslinking can improve the mechanical strength of aerogels to a certain extent, the randomly distributed and unstable crosslinking points greatly limit the improvement of mechanical properties. ${ }^{19,30}$ Moreover, low decomposition temperature $\left(<150{ }^{\circ} \mathrm{C}\right)$ of polymer-based aerogels easily causes structural collapse at high temperatures, and a few present ceramic nanofibrous aerogels are robust heat-resistant but suffer from stubborn brittleness with little deformation. Therefore, the challenge remains for constructing light-weight and thermostable nanofibrous sound-absorbing aerogels with good structural stability and full-frequency noise reduction.

Herein, we demonstrate a robust and facile strategy to create flexible ceramic nanofibrous aerogels (GCNAs) with hierarchically entangled graphene networks by integrating an in-situ co-assembly technique and a green reduction approach. Attributing to the unique hierarchically entangled structure composed of flexible ceramic nanofiber and reduced graphene oxide ( $\mathrm{rGO}$ ), the obtained GCNAs present integrated features of ultralow bulk density $\left(2 \mathrm{mg} \mathrm{cm}^{-3}\right)$, temperature-invariant superelasticity, good bendability, and desired thermal stability. Moreover, the sandwiched lamellar architectures in the thickness direction of GCNAs enable a versatile multi-interface reflection behavior, thus achieving good full-frequency sound absorption performances (noise reduction coefficient of 0.56 ). Besides, the green reduction process also endows the GCNAs with enhanced moisture insulation (water contact angle of $143^{\circ}$ ). We anticipate that the successful synthesis of the sandwiched GCNAs could break the bottlenecks of the narrow absorption band faced by traditional sound absorbers, thereby providing a new vision for developing high-efficiency traffic noise reduction materials.

\section{Results}

Fabrication and hierarchically entangled structure of flexible GCNAs. To meet the requirements of superior noise reduction property, desired structural stability, and good thermal endurance of traffic noiseabsorbing materials, we prepared flexible GCNAs in consideration of the following four demands: 1) There should be well-interconnected opened channels parallel to the direction of acoustic waves and closed-cell wall perpendicular to the direction of sound waves in GCNAs, which not only ensure the dissipation of sound waves but also prevent the sound wave from transmission through the materials. 2) The GCNAs should possess robust mechanical performance to guarantee their long-term application stability. 3) The GCNAs must have good thermal endurance to ensure safety in the high-temperature environment; meanwhile, the materials should be lightweight to reduce the energy consumption of vehicles. 4) The GCNAs should have a constantly variable assembly architecture in the thickness 
direction to achieve multi-interface reflection and gradually consume the full-frequency sound wave. To satisfy the first three requirements, flexible $\mathrm{SiO}_{2}$ nanofibers (SNFs) with good thermal stability were chosen as the building block to assemble fibrous framework structure; meanwhile, 2D GO nanosheets with good flexibility were selected as binder and macropore blocking agent to build effective entanglement among SNFs and block the pores of the fiber cavity wall. ${ }^{20,31}$ The last requirement was satisfied by designing sandwiched assembly architecture to enable multiple dissipations for fullfrequency sound waves.

The synthetic process of flexible GCNAs mainly involved four components: GO, SNFs, ascorbic acid $\left(\mathrm{C}_{6} \mathrm{H}_{8} \mathrm{O}_{6}\right)$, and ultrapure water, as presented in Fig. 1a. Above all, the flexible SNFs were prepared by versatile sol-gel electrospinning technology, which possessed uniform surface morphology with an average diameter of $286 \mathrm{~nm}$ (Supplementary Fig. 1). Upon homogenization in water, the SNFs became well-dispersed with the average fiber length of $161 \mu \mathrm{m}$ (Supplementary Fig. 2), and the obtained SNFs dispersion was mixed with $\mathrm{GO}$ aqueous solution by high-speed stirring. Then, $\mathrm{C}_{6} \mathrm{H}_{8} \mathrm{O}_{6}$ was added as a reducing agent to the above GO/SNFs dispersion for further homogenization. Subsequently, the homogenized dispersion was directional frozen in a liquid nitrogen bath and freeze-dried into GO/SNFs aerogels. ${ }^{32}$ The freshly prepared GO/SNFs aerogels were hydrophilic due to the presence of hydrophilic groups of GO (Supplementary Fig. 3a). Finally, the obtained GO/SNFs aerogels were heated at $90{ }^{\circ} \mathrm{C}$ for 6 $\mathrm{h}$ to achieve the flexible GCNAs with good hydrophobicity (Fig. $1 \mathrm{~d}$ inset and Supplementary Fig. 3b). ${ }^{33}$ Evidence of the reduction of GO/SNFs aerogels was obtained by combining the Fourier infrared (FTIR) spectra analysis, Raman spectra analysis, and X-ray diffraction spectrum (XRD) analysis. As shown in Supplementary Fig. 4a, the absorption peaks around $3300 \mathrm{~cm}^{-1}$ in the infrared spectrum of $\mathrm{GO}$ and GO/SNFs aerogels were according to the hydrophilic groups in GO, no evidence for the groups was found in GCNAs, implying the successful reduction of GO/SNFs aerogels. ${ }^{34}$ The relevant Raman spectra were shown in Supplementary Fig. $4 \mathrm{~b}$, the peak centered at $\sim 1345 \mathrm{~cm}^{-1}$ and $\sim 1583 \mathrm{~cm}^{-1}$ are corresponding to the $\mathrm{D}$ band and $\mathrm{G}$ band, which are associated with the disordered structure and the $\mathrm{sp}^{2}$-hybridized $\mathrm{C}-\mathrm{C}$ bonds, respectively. The $I_{D} / I_{G}$ of GCNAs was increased from 0.89 to 1.26 compared with GO/SNFs aerogels, indicating the formation of numerous but smaller graphitic domains after the reduction. ${ }^{35}$ Additionally, the XRD results showing in Supplementary Fig. 4c illustrated the invisibility of the GO characteristic peak at about $10.36^{\circ}$ in the GCNAs, further suggesting that the GO was successfully reduced to $\mathrm{rGO}^{36}$

The basic entangled structure of the flexible GCNAs consisted of one-dimensional (1D) flexible SNFs wrapped by 2D rGO networks (the enlarged detail of GCNAs in Fig. 1a). The unique entangled structure was comprised of multiform physical crosslinking networks, mainly including bridging, soldering, and jointing (Fig. 1b). The crosslinking network was attributed to strong $\pi-\pi$ attraction among the rGO nanosheets and van der Waals force formed between the rGO nanosheets and SNFs (Fig. 1C), playing a key role in the construction of flexible GCNAs. ${ }^{37,38}$ To provide insight into the crosslinking mechanism, the high-resolution X-ray photoelectron spectroscopy (XPS) of 0 1s peak in the interface of rGO and SNFs 
were further characterized (Supplementary Fig. 4d), which showed a distinguishable peak of C-O-Si in $531.9 \mathrm{eV}$, demonstrating the possibility of chemical bonds between $\mathrm{GO}$ and $\mathrm{SiO}_{2}$ except for the van der Waals force. ${ }^{39-41}$ Notably, we can easily adjust the density of GCNAs by adjusting the precursor GO/SNFs dispersion. Typically, the GCNAs with a density of 5,8 , and $18 \mathrm{mg} \mathrm{cm}^{-3}$ were named GCNA-5, GCNA-8, and GCNA-18, respectively. To guarantee the good formability and desired structural stability of the aerogels, the GCNAs with different weight content of GO (0 wt\%, $10 \mathrm{wt} \%, 40 \mathrm{wt} \%, 70 \mathrm{wt} \%$, and $100 \mathrm{wt} \%$ relatives to SNFs, respectively) were prepared and noted as GCNA0, GCNA10, GCNA40, GCNA70, and GCNA100, respectively, and the content of SNFs remained constant. As shown in Supplementary Fig. 5a, the mechanical performances of the GCNAs were closely related to the content of GO, we discovered that the GCNAs with 70 wt\% of GO could ensure desired structural stability. Except for special notes, all structure and performance characterizations were performed using GCNA-8 with 70 wt $\%$ GO.

Different from the common sol-gel method to prepare ceramic sound-absorbing aerogels, our fabrication process combined the simple preparation of the SNFs with the feasibility of the freeze-drying technique, which enabled rapid preparation and good structural tunability of aerogels. Fig. $1 \mathrm{~d}$ indicated that a piece of $3 \mathrm{~cm}^{3}$ flexible GCNA $\left(2 \mathrm{mg} \mathrm{cm}^{-3}\right)$ can easily stand on the tip of a feather, confirming the lightweight property. In sharp comparison with the beaded chain structure of common ceramic sound-absorbing aerogel, our directional freeze-drying process allowed the GO and SNFs to co-assemble into unique hierarchically entangled structures. As shown in the scanning electron microscopy (SEM) images of Fig. $1 \mathrm{~b}$, e-f and Supplementary Fig. 6, the hierarchical structures of flexible GCNAs consist of the opened cells (size of 50 to $100 \mu \mathrm{m}$ ), closed-cell walls (thickness of 0.5 to $2 \mu \mathrm{m}$ ), and entangled networks. The stable entangled structures were also confirmed by the energy-dispersive spectroscopy (EDS) mapping (Fig. 1g), the $\mathrm{Si}$ and $\mathrm{O}$ elements were completely covered with the $\mathrm{C}$ element, indicating that $\mathrm{rGO}$ was uniformly coated on the surface of the SNFs. The formation mechanism of the hierarchically entangled structures can be attributed to the phase transformation of the solvent and the co-assembly of SNFs and GO in the freeze-drying process (Fig. 1a, Supplementary Fig. 7, and Supplementary Discussions). ${ }^{42,43}$ The solvent water solidified into ice by liquid nitrogen and formed directional ice crystals during the bottom-up freezing process, both the nanofibers and GO nanosheets were crowded out by the front end of the ice crystal growth. Simultaneously, the aggregated GO sheets were assembled into a hierarchically entangled GO network, while SNFs were wrapped in the network to form 3D entangled structures. The macropores in the flexible GCNAs were formed by the sublimation of ice crystals during the following freezing-drying process, while the SNFs were entangled by GO networks into closed-cell walls.

Characterization of mechanical performances of GCNAs. In stark contrast to the poor bending properties of existing ceramic fibrous aerogels, the unique entangled structure endowed GCNAs with desired buckling performance, which can withstand large bending deformation without breaking (Fig. 2a inset and Supplementary Movie 1). The buckling stress-strain ( $\sigma \varepsilon \varepsilon$ ) curves (Fig. 2a) of GCNAs under different strains presented the complete closed-loop, indicating the good buckling-recovery ability of GCNAs. Moreover, the maximum buckling stress under $80 \%$ strain was $2.14 \mathrm{kPa}$, superior to other ceramic nanofibrous aerogels at the same densities. ${ }^{44}$ Furthermore, the bendable GCNAs also presented good 
cycle buckling performance. As shown in Fig. 2b, no significant decrease in max stress was discovered for the GCNAs after 1000 cyclic buckling, which can retain over $70 \%$ of the initial maximum stress (Supplementary Fig. 8), highlighting the good buckling resistance. To our surprise, compared with the hard and fragile features of common ceramic sound-absorbing aerogels, the GCNAs presented outstanding compressive performances, which can withstand large strain without breaking (Fig. 2c inset and Supplementary Movie 2). Fig. 2c showed the compressive $\sigma-\varepsilon$ curves of GCNAs under different strains, which exhibited typical three-stage deformation usually found in honeycomb-like materials: ${ }^{45}$ the linear elastic deformation zone of $\varepsilon<6 \%$, the platform zone of $6 \%<\varepsilon<55 \%$, and the densification zone of $\varepsilon>55 \%$, which were mainly caused by the elastic bending, elastic buckling, and densification of the cavity wall, respectively. ${ }^{46}$ When the $\varepsilon$ reached $80 \%$, the maximum $\sigma$ of the GCNAs could reach $17.2 \mathrm{kPa}$, which is higher than the same type of aerogels, ${ }^{20,42,43}$ indicating that the GCNAs possessed good impact resistance. Moreover, the GCNAs also showed desired compression fatigue resistance, as shown in Fig. $2 \mathrm{~d}$, the plastic deformation of the GCNAs was only $4.3 \%$ after 1000 compressions under a large strain (60\%). Besides, Young's modulus, energy dissipation coefficient, and maximum stress could remain more than $60 \%$ after 1000 cycles (Supplementary Fig. 5b), further confirming the good structural stability.

Additionally, the GCNAs could remain a stable Poisson's ratio nearly close to $0(-0.05 \sim 0)$ during the high compression and recovery process (maximum $\varepsilon$ of $80 \%$ ) (Supplementary Fig. $5 \mathrm{C}$ and 9), which is superior to most existing cellular aerogels $(>0.2) .{ }^{47,48}$ Furthermore, the GCNAs also showed remarkable dynamic mechanical stability (Supplementary Fig. 5d), the storage modulus, loss modulus, and damping ratio of the GCNAs nearly remained stable when the compression frequency increased from 0.1 to $10 \mathrm{~Hz}$, indicating the good resilience performance of the GCNAs. ${ }^{49}$ To gain insight into the good mechanical properties of GCNAs, we observed the deformation behavior of the aerogel cavity wall under the action of external force by in-situ SEM. As shown in Fig. $2 \mathrm{e}$ and $\mathrm{f}$, when the compressive strain increased from 0 to $60 \%$, the lamellar cell walls and interlamellar SNFs were gradually bent and buckled, respectively. Interestingly, the rGO nanosheets and SNFs were not found to break during compression. Subsequently, the curved cell walls and SNFs completely returned to their initial position after the strain was eliminated, further highlighting the robust structure of the GCNAs. ${ }^{38,50,51}$ Besides, Fig. $2 \mathrm{~g}$ showed the relationship between the relative Young's modulus $\left(E / E_{s}\right)$ of various aerogels and the relative bulk density $\left(\rho / \rho_{s}\right)$. Different from the low-stress transfer efficiency of random structural inorganic aerogels (the relation index of $E / E_{s}$ versus $\left.\rho / \rho_{s}>2.5\right),{ }^{20,46,52-54}$ the relationship of the GCNAs was $E / E_{s} \sim \rho / \rho_{s}{ }^{0.7}$, indicating that the GCNAs had similar elastic behavior as the low-density open-cell structured material; Meanwhile, the lamellar cavity wall of GCNAs could be bent reversibly under the action of external force, so the loading stress could be effectively transferred among the cavity walls of the aerogel. More interestingly, compared with the little tensile performance of existing ceramic fiber aerogels, the unique hierarchically entangled structures also endow GCNAs with certain stretchable properties (Fig. 2h). The tensile stress firstly improved linearly with the increase of tensile strain, and Young's modulus reaching $282 \mathrm{kPa}$. Further increase in tensile strain resulted in the gradual fracture of the GCNAs, meanwhile, the tensile stress and strain were $12.56 \mathrm{kPa}$ and $4.8 \%$, respectively. ${ }^{20,50}$ 
Current sound-absorbing materials are mainly made of polymer, which has good elasticity at room temperature but can't use at high temperatures. In contrast, ceramic materials possess good heat resistance but suffer from stubborn brittleness with little deformation. Our flexible GCNAs combined the structural characteristics of polymer materials and the high-temperature resistance of ceramics, which still exhibit good elasticity at high temperatures. As shown in Fig. 2i-k, the GCNAs exhibited stable viscoelasticity at different ambient temperatures, the storage modulus, loss modulus, and damping ratio almost remained at stable values in $-100 \sim 500^{\circ} \mathrm{C} .{ }^{55}$ Moreover, the relationship of dynamic mechanical performances of the GCNAs versus continuously changing temperature was further investigated, as shown in Fig. 2l, the storage modulus, loss modulus, and damping ratio of the GCNAs remained unchanged with the ambient temperature continuously changing from $-100 \sim 500{ }^{\circ} \mathrm{C}$, indicating that the GCNAs can be used for noise absorption in extreme temperatures. ${ }^{56}$ Evidence of good thermal endurance of GCNAs also came from thermogravimetry analysis (TGA), as shown in Supplementary Fig. 10, the TGA plot of GCNAs showed a significant weight loss only when the temperature above $500{ }^{\circ} \mathrm{C}$. Additionally, no structural collapse and ignition were observed when the GCNAs were compressed in a high-temperature flame $\left(\sim 550^{\circ} \mathrm{C}\right)$ with a large strain (Supplementary Fig. 11), highlighting that the GCNAs still had superelasticity at high temperature. Besides, the GCNAs almost had no plastic deformation after being compressed in liquid nitrogen with a large strain of $80 \%$ (Supplementary Fig. 12 and Movie 3), confirming the superelasticity of GCNAs under low-temperature.

Sound absorption performances of flexible GCNAs. Taking into consideration the hierarchically entangled structures, desired mechanical properties, and good temperature resistance, the flexible GCNAs show great application potential in noise absorption. Generally, the sound absorption performances were decided by the assembly structure, which was mainly influenced by the GO loading amounts (10 100 $w t \%)$. As presented in Fig. 3a-d, the coverage area of rGO networks on nanofibrous cell walls gradually increased with the improvement of $\mathrm{GO}$ loading amounts, resulting in the reduction of connectivity of cell walls within GCNAs. Moreover, the airflow resistance of GCNAs was greatly improved from $1.2 \times 10^{5}$ to $6.7 \times 10^{5} \mathrm{~Pa} \mathrm{~s} \mathrm{~m}^{-2}$ with increasing GO loading amount (10 70 wt\%), indicating that the GO networks effectively optimized the pore structure of the GCNAs (Fig. 3l). However, further increasing GO loading amount decreased the airflow resistance, which was because that excess $\mathrm{GO}$ agglomerated and unevenly distributed caused by the strong $\pi-\pi$ attraction (Fig. 3d). Noise reduction coefficient (NRC), the average value of absorption coefficients in $250,500,1000$, and $2000 \mathrm{~Hz}$, is usually used to appraise the noise absorption materials. ${ }^{11,12,27}$ The NRC of the GCNAs with increasing GO loading amounts were $0.19,0.21$, 0.27 , and 0.26 , respectively (Fig. $31-\mathrm{m}$ ), which was consistent with flow resistance.

Additionally, the GCNAs could remain a stable Poisson's ratio nearly close to $0(-0.05 \sim 0)$ during the high compression and recovery process (maximum $\varepsilon$ of $80 \%$ ) (Supplementary Fig. $5 \mathrm{C}$ and 9), which is superior to most existing cellular aerogels (>0.2). ${ }^{47,48}$ Furthermore, the GCNAs also showed remarkable dynamic mechanical stability (Supplementary Fig. $5 \mathrm{~d}$ ), the storage modulus, loss modulus, and damping ratio of the GCNAs nearly remained stable when the compression frequency increased from 0.1 to $10 \mathrm{~Hz}$, indicating the good resilience performance of the GCNAs. ${ }^{49}$ To gain insight into the good mechanical 
properties of GCNAs, we observed the deformation behavior of the aerogel cavity wall under the action of external force by in-situ SEM. As shown in Fig. $2 \mathrm{e}$ and $\mathrm{f}$, when the compressive strain increased from 0 to $60 \%$, the lamellar cell walls and interlamellar SNFs were gradually bent and buckled, respectively. Interestingly, the rGO nanosheets and SNFs were not found to break during compression. Subsequently, the curved cell walls and SNFs completely returned to their initial position after the strain was eliminated, further highlighting the robust structure of the GCNAs. ${ }^{38,50,51}$ Besides, Fig. $2 \mathrm{~g}$ showed the relationship between the relative Young's modulus $\left(E / E_{s}\right)$ of various aerogels and the relative bulk density $\left(\rho / \rho_{s}\right)$. Different from the low-stress transfer efficiency of random structural inorganic aerogels (the relation index of $E / E_{s}$ versus $\left.\rho / \rho_{s}>2.5\right),{ }^{20,46,52-54}$ the relationship of the GCNAs was $E / E_{s} \sim \rho / \rho_{s}{ }^{0.7}$, indicating that the GCNAs had similar elastic behavior as the low-density open-cell structured material; Meanwhile, the lamellar cavity wall of GCNAs could be bent reversibly under the action of external force, so the loading stress could be effectively transferred among the cavity walls of the aerogel. More interestingly, compared with the little tensile performance of existing ceramic fiber aerogels, the unique hierarchically entangled structures also endow GCNAs with certain stretchable properties (Fig. $2 \mathrm{~h}$ ). The tensile stress firstly improved linearly with the increase of tensile strain, and Young's modulus reaching $282 \mathrm{kPa}$. Further increase in tensile strain resulted in the gradual fracture of the GCNAs, meanwhile, the tensile stress and strain were $12.56 \mathrm{kPa}$ and $4.8 \%$, respectively. ${ }^{20,50}$

Current sound-absorbing materials are mainly made of polymer, which has good elasticity at room temperature but can't use at high temperatures. In contrast, ceramic materials possess good heat resistance but suffer from stubborn brittleness with little deformation. Our flexible GCNAs combined the structural characteristics of polymer materials and the high-temperature resistance of ceramics, which still exhibit good elasticity at high temperatures. As shown in Fig. 2i-k, the GCNAs exhibited stable viscoelasticity at different ambient temperatures, the storage modulus, loss modulus, and damping ratio almost remained at stable values in $-100 \sim 500{ }^{\circ} \mathrm{C} .{ }^{55}$ Moreover, the relationship of dynamic mechanical performances of the GCNAs versus continuously changing temperature was further investigated, as shown in Fig. 2l, the storage modulus, loss modulus, and damping ratio of the GCNAs remained unchanged with the ambient temperature continuously changing from $-100 \sim 500^{\circ} \mathrm{C}$, indicating that the GCNAs can be used for noise absorption in extreme temperatures. ${ }^{56}$ Evidence of good thermal endurance of GCNAs also came from thermogravimetry analysis (TGA), as shown in Supplementary Fig. 10, the TGA plot of GCNAs showed a significant weight loss only when the temperature above $500{ }^{\circ} \mathrm{C}$. Additionally, no structural collapse and ignition were observed when the GCNAs were compressed in a high-temperature flame $\left(\sim 550^{\circ} \mathrm{C}\right)$ with a large strain (Supplementary Fig. 11), highlighting that the GCNAs still had superelasticity at high temperature. Besides, the GCNAs almost had no plastic deformation after being compressed in liquid nitrogen with a large strain of 80\% (Supplementary Fig. 12 and Movie 3), confirming the superelasticity of GCNAs under low-temperature.

Sound absorption performances of flexible GCNAs. Taking into consideration the hierarchically entangled structures, desired mechanical properties, and good temperature resistance, the flexible GCNAs show great application potential in noise absorption. Generally, the sound absorption performances were 
decided by the assembly structure, which was mainly influenced by the GO loading amounts (10 100 $w t \%)$. As presented in Fig. 3a-d, the coverage area of rGO networks on nanofibrous cell walls gradually increased with the improvement of $\mathrm{GO}$ loading amounts, resulting in the reduction of connectivity of cell walls within GCNAs. Moreover, the airflow resistance of GCNAs was greatly improved from $1.2 \times 10^{5}$ to $6.7 \times 10^{5} \mathrm{~Pa} \mathrm{~s} \mathrm{~m}^{-2}$ with increasing GO loading amount (10 70 wt\%), indicating that the GO networks effectively optimized the pore structure of the GCNAs (Fig. 3l). However, further increasing GO loading amount decreased the airflow resistance, which was because that excess $\mathrm{GO}$ agglomerated and unevenly distributed caused by the strong $\pi-\pi$ attraction (Fig. 3d). Noise reduction coefficient (NRC), the average value of absorption coefficients in $250,500,1000$, and $2000 \mathrm{~Hz}$, is usually used to appraise the noise absorption materials. ${ }^{11,12,27}$ The NRC of the GCNAs with increasing GO loading amounts were $0.19,0.21$, 0.27 , and 0.26 , respectively (Fig. $3 \mathrm{l}-\mathrm{m}$ ), which was consistent with flow resistance.

To explore the sound absorption mechanism of the sandwich structure, the 3D structural models of GCNA-5, GCNA-18, and sandwiched GCNAs were constructed (Fig. 4e, Supplementary Fig. 15, Table 2, and Supplementary Methods). ${ }^{59}$ Obviously, the sound pressure drop of the GCNA-18 and sandwiched GCNAs (red to green color) was much greater than GCNA-5 (red to yellow color), which was consistent with the poor low-frequency absorption of GCNA-5. However, in contrast to the rapid drop in sound pressure of GCNA-18 as a consequence of its overlarge density and excessive reflections, the sound pressure of the sandwiched GCNA was generally high in the first unit and then decreased continuously, indicating that more sound waves could enter and then were gradually consumed, which were consistent with high absorption coefficients of sandwiched GCNA. Based on the above results, we further constructed the structural element models and simulated sound absorption mechanisms (Supplementary Fig. 16), the low-density GCNA-5 with macroporous structure preferred to consuming high-frequency noise (Supplementary Fig. 16a), while the high-density GCNA-18 with a small porous structure preferred to consuming low-frequency noise (Supplementary Fig. 16b). Interestingly, by designing a sandwiched architecture, an attractive full-frequency sound absorption behavior was found: the low-density and highdensity lamellar structures were responsible for effectively consuming high-frequency and low-frequency sound waves, respectively, thus successfully absorbing the full-frequency sound waves (Supplementary Fig. 16c). Besides, the sandwich structure effectively increased the internal interface of GCNAs, which enhanced the multistage reflection path of sound waves inside the material and thus successfully increased the sound energy consumption. ${ }^{9}$ To confirm the outstanding noise reduction performance of the GCNAs, the NRC and corresponding areal density of the sandwiched GCNAs were compared with typical already reported and commercial noise reduction materials ${ }^{19,27,63}$. As shown in Fig. $4 \mathrm{f}$, the sandwiched GCNAs exhibited high noise absorption (NRC of 0.56$)$ while ultralight property $\left(280.8 \mathrm{~g} \mathrm{~m}^{-2}\right)$ in contrast to present noise absorbers, which was because that the sandwich structure increased the contact area of sound waves, providing more frictional resistance for the acoustic energy; meanwhile, the resonance of the cavity wall also dissipated the acoustic energy. ${ }^{8}$

As a proof of concept for traffic noise reduction applications, the white noise of $\sim 80 \mathrm{~dB}$ was generated by a loudspeaker within a well-sealed pipe to mimic traffic noise, after passing commercial fiber felt at the 
middle of the pipe, the noise only decreased to $67.4 \mathrm{~dB}$, due to the large fiber diameter of $>5 \mu \mathrm{m}$, the large density of $17 \mathrm{mg} \mathrm{cm}^{-3}$, and open-cell walls; In dramatic contrast, our sandwiched GCNAs could sharply decrease noise to $53.4 \mathrm{~dB}$ by their closed-cell walls (Fig. 4g), highlighting the great practical application performance of the sandwiched GCNAs. We further monitored the absorption capacity of real automobile engines noise, as shown in Fig. 4h and Supplementary Fig. 17a, a bottom sealed round pipe loading with commercial fiber felt and our sandwiched GCNAs were placed next to a working engine, the sandwiched GCNAs reduced noise by $19.4 \mathrm{~dB}(95.5$ to $76.1 \mathrm{~dB}$ ), whereas the fiber felt only reduced by $8.3 \mathrm{~dB}$ (95.5 to 87.2 dB). Moreover, the sandwiched GCNAs exhibited promising noise absorption against running air compressor in high temperature, as shown in Supplementary Fig. 17b, the sandwiched GCNAs reduced noise by $19 \mathrm{~dB}$ ( 94.2 to $75.2 \mathrm{~dB}$ ), whereas the commercial fiber felt only reduced by $10.1 \mathrm{~dB}$ (94.2 to 84.1 $\mathrm{dB}$ ). Furthermore, when a heating stage with a temperature of $250^{\circ} \mathrm{C}$ was close to two samples, the sandwiched GCNAs not only reduced noise by $17.7 \mathrm{~dB}$ after 10 min but also maintain intact morphology, whereas the fiber felt was significantly decomposed and shrunk, with almost no sound absorption (92.4 to $90.2 \mathrm{~dB}$ ) (Fig. 4i and Supplementary Fig. 17c). These results indicate that the sandwiched GCNAs could serve as robust noise-absorbing materials with potentially broad applications, such as traffic noise reduction, industrial noise reduction, and domestic noise reduction, especially for safety noise reduction in high-temperature environments.

\section{Discussion}

The design and construction of GCNAs open a new path for searching the advanced applications of 1D ceramic nanofibers in 3D macrostructures. In this study, flexible ceramic nanofibrous aerogels were served as a practical verification of the conceptual model; in consideration of the rich source of ceramic material as well as the facile preparation of ceramic nanofibers, our research will provide new ideas for novel ceramic nanofibrous aerogels for application in various fields. For example, bioceramics (such as $\mathrm{ZrO}_{2}$ and hydroxyapatite) have been widely used as bone tissue substitutes; thus, creating 3D flexible ceramic nanofibrous aerogels using these bioceramic nanofibers can more really simulate bone tissue by providing suitable direction for cell growth. ${ }^{64}$ Moreover, honeycomb ceramics (such as $\mathrm{Al}_{2} \mathrm{O}_{3}$ and $\mathrm{TiO}_{2}$ ) are usually used as catalyst carriers but suffer from stubborn brittleness, the ceramic nanofibrous aerogels not only possess robust flexibility but also enable to load of more nano-catalysts, which could significantly improve the application performance. Additionally, some functional materials (such as $\mathrm{Au}$, $\mathrm{Fe}_{3} \mathrm{O}_{4}$, and other nanoparticles) could be easily embedded in the open space of GCNAs, enabling the fabrication of various new composite aerogels.

In summary, we presented a robust methodology to construct flexible ceramic nanofibrous aerogels with hierarchically entangled graphene networks. Benefiting from the entangled structure composed of rGO networks wrapped ceramic nanofiber, the GCNAs achieved ultralight properties, temperature-invariant superelasticity (4.3\% plastic deformation at the 1000th cycle), and good bendability (unchanged structures over 1000 times). Furthermore, the hierarchically entangled sandwich structures enable a versatile multi-interface reflection capability, contributing to the striking sound absorption performance 
(NRC of 0.56). Moreover, the comprehensive advantages of ceramic and rGO endowed GCNAs with good heat resistance and enhanced moisture insulation. We envision that these exceptional sandwiched GCNAs that can be easily scaled up will pave the way for new types of sound absorption materials used for removing full-frequency noise, involving traffic noise reduction, industrial noise reduction, and domestic noise reduction.

\section{Methods}

Methods are available in the Supporting Information.

\section{Declarations}

\section{Data availability}

The experimental data that support the findings of this study are available from the corresponding author upon reasonable request.

\section{Acknowledgments}

This work was supported by the Natural Science Foundation of China (No. 51925302 and 51873031), the Science and Technology Commission of Shanghai Municipality (No. 20QA1400500), and the Fundamental Research Funds for the Central Universities (No. LZB2019004 and CUSF-DH-D-2020040).

\section{Author contributions}

B.D., S.Z., and D.Z. designed the research and wrote the manuscript. D.Z. and J.Y. involved the analysis of performance data. L.C., D.Z., X.Y., and Y.S. prepared the samples and performed structural analysis. D.Z. and L.C. performed the sound absorption tests and other property characterizations.

\section{Additional information}

Competing interests: The authors declare no competing interests.

\section{References}

1 Sorensen, M. \& Pershagen, G. Transportation noise linked to cardiovascular disease independent from air pollution. Eur. Heart J. 40, 604-606 (2019).

2 Sohrabi, S. \& Khreis, H. Burden of disease from transportation noise and motor vehicle crashes: Analysis of data from houston, texas. Environ. Int. 136, 105520 (2020).

3 Chadha, S., Lopez, L., Agarwal, P. \& Ambett, R. Global costs of unaddressed hearing loss and costseffectiveness of interventions. A WHO Report (2017). 
4 Kalauni, K. \& Pawar, S. J. A review on the taxonomy, factors associated with sound absorption and theoretical modeling of porous sound absorbing materials. J. Porous Mat. 26, 1795-1819 (2019).

5 Dunne, R., Desai, D. \& Sadiku, R. A review of the factors that influence sound absorption and the available empirical models for fibrous materials. Acoust. Aust. 45, 453-469 (2017).

6 Soltani, P., Azimian, M., Wiegmann, A. \& Zarrebini, M. Experimental and computational analysis of sound absorption behavior in needled nonwovens. J. Sound Vib. 426, 1-18 (2018).

7 Tang, X. N. \& Yan, X. Acoustic energy absorption properties of fibrous materials: A review. Compos. Part A 101, 360-380 (2017).

8 Cao, L. T., Fu, Q. X., Si, Y., Ding, B. \& Yu, J. Y. Porous materials for sound absorption. Compos. Commun. 10, 25-35 (2018).

9 Zong, D. et al. Interlocked dual-network and superelastic electrospun fibrous sponges for efficient low-frequency noise absorption. Small Str. 1, 2000004 (2020).

10 Lyu, L. et al. Sound absorption properties of multi-layer structural composite materials based on waste corn husk fibers. J. Eng. Fiber. Fabr. 15, 1-8 (2020).

11 Cao, L., Si, Y., Yin, X., Yu, J. \& Ding, B. Ultralight and resilient electrospun fiber sponge with a lamellar corrugated microstructure for effective low-frequency sound absorption. ACS Appl. Mater. Inter. 11, 35333-35342 (2019).

$12 \mathrm{Jia}, \mathrm{C}$. et al. Highly compressible and anisotropic lamellar ceramic sponges with superior thermal insulation and acoustic absorption performances. Nat. Commun. 11, 3732 (2020).

13 Nine, M. J. et al. Graphene oxide-based lamella network for enhanced sound absorption. Adv. Funct. Mater. 27, 1703820 (2017).

14 Pinto, S. C. et al. Multifunctional hybrid structures made of open-cell aluminum foam impregnated with cellulose/graphene nanocomposites. Carbohyd. Polym. 238, 116197 (2020).

15 Lu, B. et al. High performance broadband acoustic absorption and sound sensing of a bubbled graphene monolith. J. Mater. Chem. A 7, 11423-11429 (2019).

16 Liu, L. et al. A graphene oxide and functionalized carbon nanotube based semi-open cellular network for sound absorption. Soft Matter 15, 2269-2276 (2019).

17 Zhang, S. et al. Highly efficient, transparent, and multifunctional air filters using self-assembled 2D nanoarchitectured fibrous networks. ACS Nano 13, 13501-13512 (2019). 
18 Zhang, S. et al. Spider-web-inspired $\mathrm{PM}_{0.3}$ filters based on self-sustained electrostatic nanostructured networks. Adv. Mater. 32, 2002361 (2020).

19 Si, Y., Yu, J., Tang, X., Ge, J. \& Ding, B. Ultralight nanofibre-assembled cellular aerogels with superelasticity and multifunctionality. Nat. Commun. 5, 5802 (2014).

$20 \mathrm{Si}$, Y., Wang, X., Dou, L., Yu, J. \& Ding, B. Ultralight and fire-resistant ceramic nanofibrous aerogels with temperature-invariant superelasticity. Sci. Adv. 4, eaas8925 (2018).

21 Zhang, S. et al. Direct electronetting of high-performance membranes based on self-assembled 2D nanoarchitectured networks. Nat. Commun. 10, 1458 (2019).

22 Zhang, S., Liu, H., Yu, J., Li, B. \& Ding, B. Multi-functional flexible 2D carbon nanostructured networks. Nat. Commun. 11, 5134 (2020).

23 Ding, J. et al. Electrospun polymer biomaterials. Prog. Polym. Sci. 90, 1-34 (2019).

24 Ding, Y. et al. Electrospun fibrous architectures for drug delivery, tissue engineering and cancer therapy. Adv. Funct. Mater. 29, 1802852 (2019).

25 Jiang, S., Agarwal, S. \& Greiner, A. Low-density open cellular sponges as functional materials. Angew. Chem. Int. Ed. 56, 15520-15538 (2017).

26 Xue, J., Wu, T., Dai, Y. \& Xia, Y. Electrospinning and electrospun nanofibers: Methods, materials, and applications. Chem. Rev. 119, 5298-5415 (2019).

27 Cao, L. et al. Ultralight, superelastic and bendable lashing-structured nanofibrous aerogels for effective sound absorption. Nanoscale 11, 2289-2298 (2019).

$28 \mathrm{He}, \mathrm{C}$. et al. Mechanically resistant and sustainable cellulose-based composite aerogels with excellent flame retardant, sound-absorption, and superantiwetting ability for advanced engineering materials. ACS Sustain. Chem. Eng. 6, 927-936 (2018).

29 Wang, C. et al. Cellulose as an adhesion agent for the synthesis of lignin aerogel with strong mechanical performance, sound-absorption and thermal insulation. Sci. Rep.-UK 6, 32383 (2016).

30 Dong, X., Cao, L., Si, Y., Ding, B. \& Deng, H. Cellular structured CNTs@SiO 2 nanofibrous aerogels with vertically aligned vessels for salt-resistant solar desalination. Adv. Mater. 32, 1908269 (2020).

31 Shabangoli, Y. et al. Nile blue functionalized graphene aerogel as a pseudocapacitive negative electrode material across the full PH range. ACS Nano 13, 12567-12576 (2019).

32 Wang, C. H. et al. Freeze-casting produces a graphene oxide aerogel with a radial and centrosymmetric structure. ACS Nano 12, 5816-5825 (2018). 
33 Kim, Y. et al. Star-shaped Pd@Pt core-shell catalysts supported on reduced graphene oxide with superior electrocatalytic performance. J. Mater. Chem. A 2, 6976-6986 (2014).

34 Zhou, L. J. \& Xu, Z. Y. Ultralight, highly compressible, hydrophobic and anisotropic lamellar carbon aerogels from graphene/polyvinyl alcohol/cellulose nanofiber aerogel as oil removing absorbents. J. Hazard. Mater. 388, 121804 (2020).

35 Jiang, Y. Q. et al. Direct 3D printing of ultralight graphene oxide aerogel microlattices. Adv. Funct. Mater. 28, 1707024 (2018).

36 Cao, X., Zhang, J., Chen, S., Varley, R. J. \& Pan, K. 1D/2D nanomaterials synergistic, compressible, and response rapidly 3D graphene aerogel for piezoresistive sensor. Adv. Funct. Mater. 30, 2003618 (2020).

37 Sun, H., Xu, Z. \& Gao, C. Multifunctional, ultra-flyweight, synergistically assembled carbon aerogels. Adv. Mater. 25, 2554-2560 (2013).

$38 \mathrm{Xu}, \mathrm{X}$. et al. Naturally dried graphene aerogels with superelasticity and tunable poisson's ratio. Adv. Mater. 28, 9223-9230 (2016).

39 Yang, L. et al. Graphene oxide glue-electrode for fabrication of vertical, elastic, conductive columns. ACS Nano 11, 2944-2951 (2017).

40 Ma, Q., Zhu, X., Zhang, D. \& Liu, S. Graphene oxide - a surprisingly good nucleation seed and adhesion promotion agent for one-step $\mathrm{ZnO}$ lithography and optoelectronic applications. J. Mater. Chem. C2, 8956-8961 (2014).

41 Xia, Q. et al. Phenyl tris-2-methoxydiethoxy silane as an additive to PC-based electrolytes for lithium-ion batteries. J. Power Sources 180, 602-606 (2008).

$42 \mathrm{Si}$, Y. et al. Ultralight biomass-derived carbonaceous nanofibrous aerogels with superelasticity and high pressure-sensitivity. Adv. Mater. 28, 9512-9518 (2016).

$43 \mathrm{Si}$, Y. et al. Ultrahigh-water-content, superelastic, and shape-memory nanofiber-assembled hydrogels exhibiting pressure-responsive conductivity. Adv. Mater. 29, 1700339 (2017).

44 Dou, L. et al. Interweaved cellular structured ceramic nanofibrous aerogels with superior bendability and compressibility. Adv. Funct. Mater. 30, 2005928 (2020).

$45 \mathrm{Kim}, \mathrm{K}$. H., Oh, Y. \& Islam, M. F. Graphene coating makes carbon nanotube aerogels superelastic and resistant to fatigue. Nat. Nanotechnol. 7, 562-566 (2012).

46 Qiu, L., Liu, J. Z., Chang, S. L. Y., Wu, Y. \& Li, D. Biomimetic superelastic graphene-based cellular monoliths. Nat. Commun. 3, 1241 (2012). 

tetrakaidecahedral unit cell and Castigliano's second theorem. Compos. Sci. Technol. 63, 1769-1781 (2003).

48 Fu, Q. et al. Highly carboxylated, cellular structured, and underwater superelastic nanofibrous aerogels for efficient protein separation. Adv. Funct. Mater. 29, 1808234 (2019).

49 Wang, F. et al. Biomimetic and superelastic silica nanofibrous aerogels with rechargeable bactericidal function for antifouling water disinfection. ACS Nano 14, 8975-8984 (2020).

50 Guo, F. et al. Millisecond response of shape memory polymer nanocomposite aerogel powered by stretchable graphene framework. ACS Nano 13, 5549-5558 (2019).

51 Ma, Y. N. et al. 3D synergistical MXene/reduced graphene oxide aerogel for a piezoresistive sensor. ACS Nano 12, 3209-3216 (2018).

52 Pekala, R. W., Alviso, C. T. \& Lemay, J. D. Organic aerogels: microstructural dependence of mechanical properties in compression. J. Non-Cryst. Solids 125, 67-75 (1990).

53 Zheng, X. et al. Ultralight, ultrastiff mechanical metamaterials. Science 344, 1373-1377 (2014).

54 Worsley, M. A., Kucheyev, S. O., Satcher, J. H., Hamza, A. V. \& Baumann, T. F. Mechanically robust and electrically conductive carbon nanotube foams. Appl. Phys. Lett. 94, 073115 (2009).

55 Wang, F. et al. In situ synthesis of biomimetic silica nanofibrous aerogels with temperatureinvariant superelasticity over one million compressions. Angew. Chem. Int. Ed. 59, 8285-8292 (2020).

56 Zhang, X. et al. Ultrastrong, superelastic, and lamellar multiarch structured $\mathrm{ZrO}_{2}-\mathrm{Al}_{2} \mathrm{O}_{3}$ nanofibrous aerogels with high-temperature resistance over $1300^{\circ} \mathrm{C}$. ACS Nano 14, 15616-15625 (2020).

57 Berardi, U. \& lannace, G. Acoustic characterization of natural fibers for sound absorption applications. Build. Environ. 94, 840-852 (2015).

58 Wang, D., Peng, L. M., Fu, F., Liu, M. H. \& Song, B. Q. Analysis of polyester/wood composite fiber needling mat sound absorption performance. Polym. Composite. 39, 3823-3830 (2018).

59 Xie, S. C., Yang, S. C., Yang, C. X. \& Wang, D. Sound absorption performance of a filled honeycomb composite structure. Appl. Acoust. 162, 107202 (2020).

$60 \mathrm{Li}, \mathrm{T}$. T. et al. Sound absorption and compressive property of PU foam-filled composite sandwiches: Effects of needle-punched fabric structure, porous structure, and fabric-foam interface. Polym. Adv. Technol. 31, 451-460 (2020). 
61 Yang, X. et al. A simplistic unit cell model for sound absorption of cellular foams with fully/semiopen cells. Compos. Sci. Technol. 118, 276-283 (2015).

62 Liu, Y., Lyu, L., Guo, J. \& Wang, Y. Sound absorption performance of the poplar seed fiber/PCL composite materials. Materials 13, 1465 (2020).

63 Buratti, C., Merli, F. \& Moretti, E. Aerogel-based materials for building applications: Influence of granule size on thermal and acoustic performance. Energ. Buildings 152, 472-482 (2017).

64 Koons, G. L., Diba, M. \& Mikos, A. G. Materials design for bone-tissue engineering. Nat. Rev. Mater. 5, 584-603 (2020).

\section{Figures}

a

GOISNF dispersion

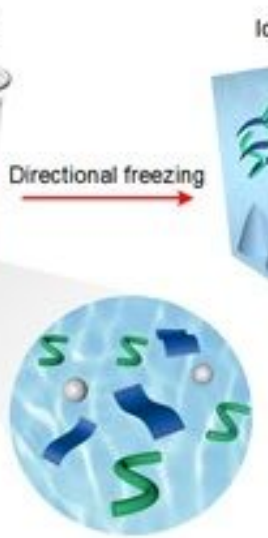

Ice crystal formation
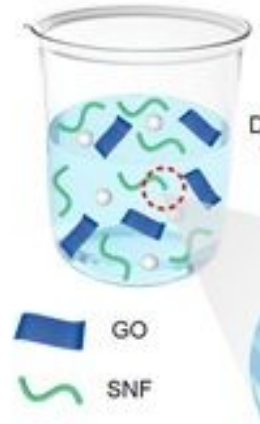

Ascorbic acid
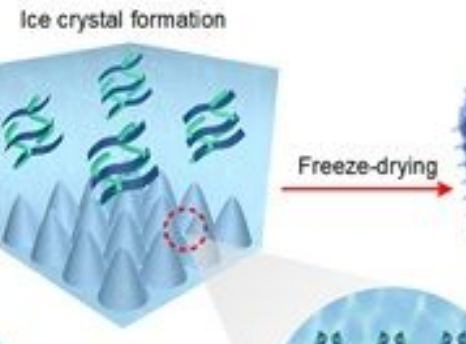

Directional
ice crystals

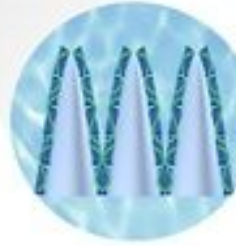

b


d

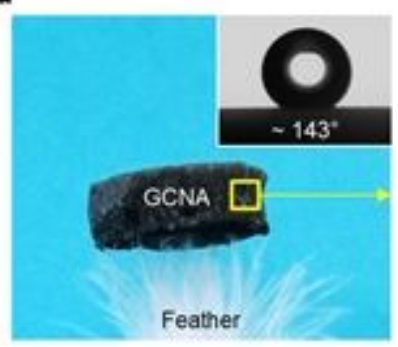

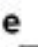

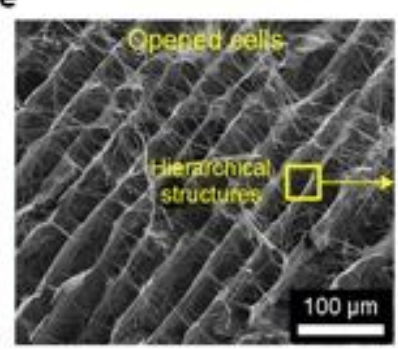

f

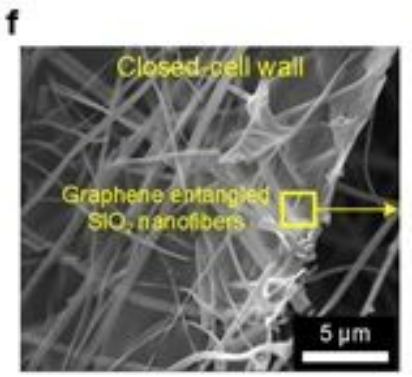

GOISNF aerogels

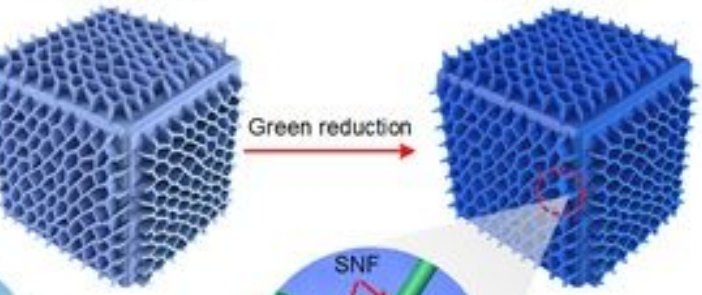

cell wall

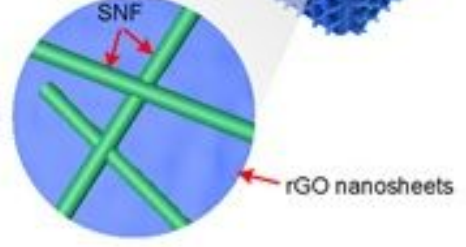

C

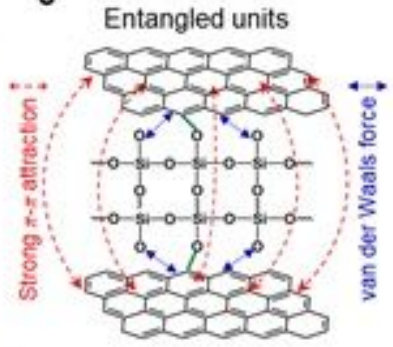

g

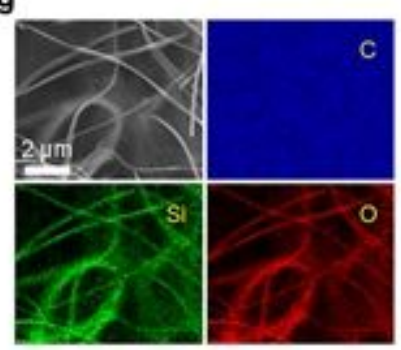

Figure 1

Structure design and hierarchical architectures of GCNAs. (a) Schematic illustration of the fabrication of flexible GCNAs. (b) Schematics and corresponding SEM images of the hierarchically entangled crosslinking networks. (c) The entangled unit of the crosslinking networks. (d) An optical image showing 
a $3 \mathrm{~cm} 3 \mathrm{GCNA}$ ( $\rho=2 \mathrm{mg} \mathrm{cm}-3$ ) standing on the tip of feathers. Insets: photograph of the water droplet on the GCNA in the air. (e-f) The microscopic structure of flexible GCNAs at different magnifications demonstrating hierarchical structure. (g) SEM-EDS images of a closed-cell wall with corresponding elemental mapping images of $\mathrm{C}, \mathrm{Si}$, and $\mathrm{O}$.
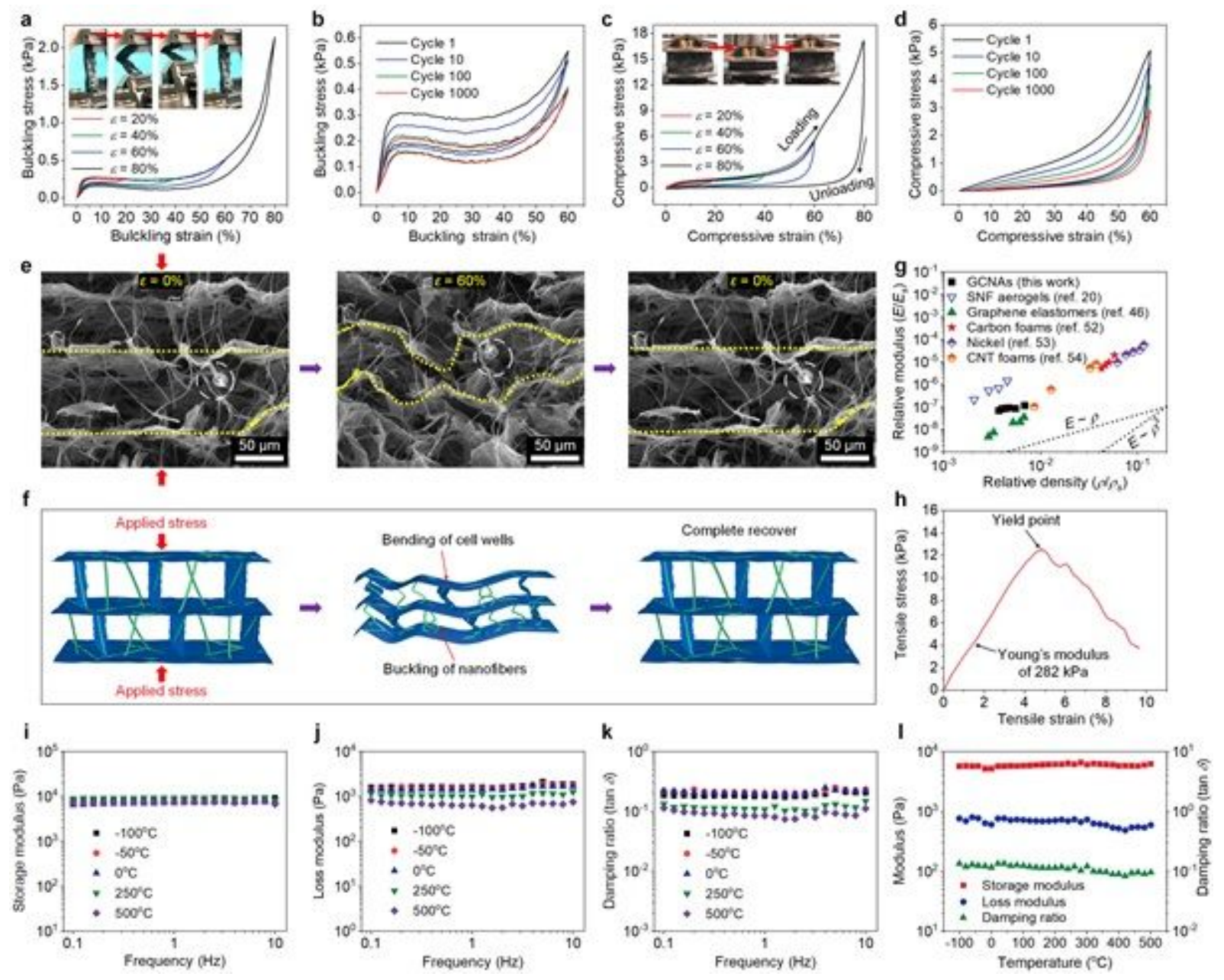

\section{Figure 2}

Mechanical properties of the GCNAs over a wide range of temperatures. (a) $\sigma-\varepsilon$ curves during bucklingrecovery cycles with increasing $\varepsilon$ amplitude; the inset shows photographs of flexible GCNAs under a buckling-recovery cycle $(\varepsilon=80 \%$ ). (b) A 1000-cycle bending fatigue test with $\varepsilon=60 \%$. (c) Compressive $\sigma$ versus $\varepsilon$ curves during loading-unloading cycles with increasing $\varepsilon$ amplitude; insets: photographs of GCNAs under a compressing and releasing cycle $(\varepsilon=60 \%$ ). (d) A 1000-cycle fatigue test with compressive $\varepsilon$ of $60 \%$. In-situ SEM observations (e) and corresponding structure diagram (f) of the GCNAs under compression and release, focusing on a small piece $(<0.3 \mathrm{~mm})$. $(\mathrm{g})$ The relative Young's modulus of selected aerogels with low densities. (h) The tensile $\sigma-\varepsilon$ curve for the GCNAs. (i-k) Storage modulus, loss modulus, and damping ratio of the GCNAs versus frequency $(0.1$ to $10 \mathrm{~Hz})$ at temperatures from -100 to $500{ }^{\circ} \mathrm{C}$, with an oscillatory $\varepsilon$ of $1 \%$. (I) The temperature dependence of the storage modulus, loss modulus, and damping ratio for GCNAs; the oscillatory $\varepsilon$ was $1 \%$ and the frequency was $1 \mathrm{~Hz}$. 
a


Figure 3

Sound absorption performances of flexible GCNAs. SEM images showing the cell wall of GCNAs with various GO loading amounts of (a) $10 \mathrm{wt} \%$, (b) $40 \mathrm{wt} \%$, (c) $70 \mathrm{wt} \%$, and (d) $100 \mathrm{wt} \%$. (e-h) The corresponding absolute sound pressure distribution of various $\mathrm{GO}$ loading amounts. Schematic illustrations of the mechanism for the sound absorption of (i) open-cell walls, (j) semi-open cell walls, and (k) closed-cell walls. (I) Influences of GO loading amount on NRC and airflow resistance of the GCNAs. (m) Variation of the absorption coefficient of the relevant GCNAs. (n) Effects of density on the sound absorption coefficient of GCNAs. (o) NRC of GCNA-5 with various thicknesses. 
a

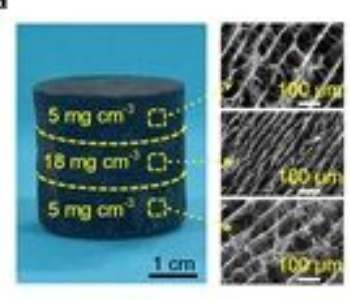

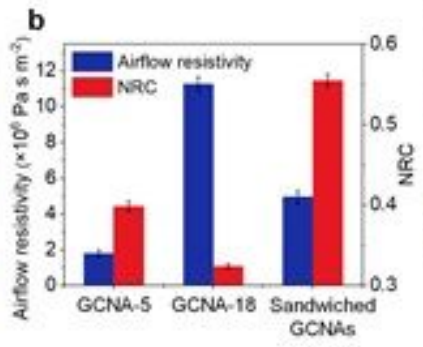
GCNAs
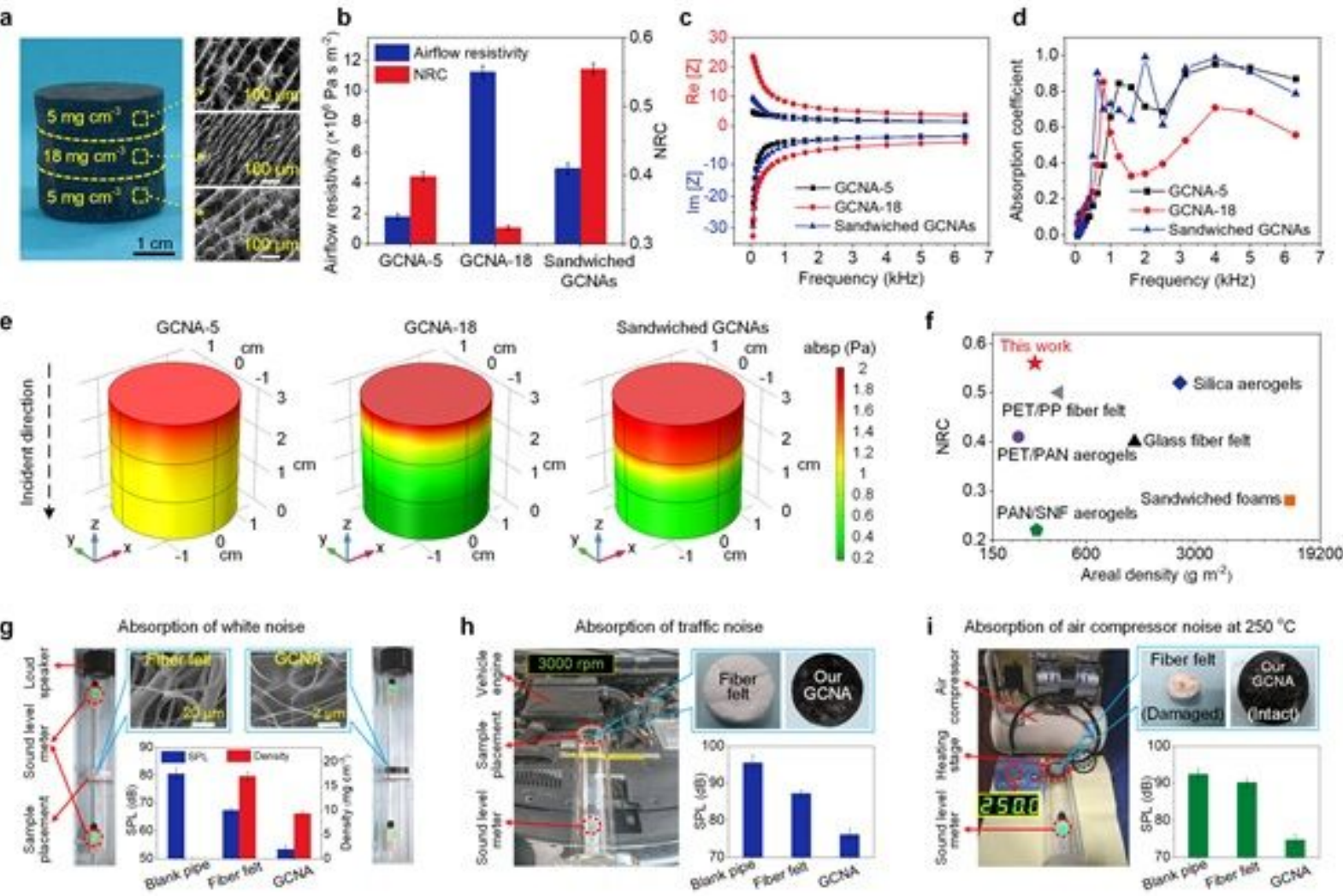

i Absorption of air compressor noise at $250^{\circ} \mathrm{C}$

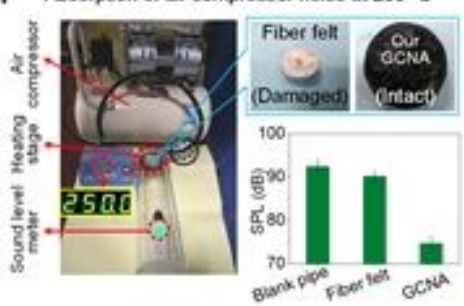

Figure 4

Sound absorption performances of sandwiched GCNAs. (a) Optical and SEM images of sandwiched GCNAs showing the macroscopic continuity and fluffy-dense-fluffy microscopic structure. (b) Airflow resistivity and NRC of GCNA-5, GCNA-18, and sandwiched GCNA. The specific surface acoustic impedance (c) and sound absorption coefficients (d) of GCNA-5, GCNA-18, and sandwiched GCNA. (e) Absolute sound pressure distribution of GCNA-5, GCNA-18, and sandwiched GCNA in $500 \mathrm{~Hz}$. (f) Comparison of NRC for the different noise reduction materials and the sandwiched GCNAs. Photographs showing the direct application of the commercial fiber felt and sandwiched GCNAs on the $(\mathrm{g})$ white noise absorption, (h) automobile engine noise absorption at $3000 \mathrm{rpm}$, and (i) air compressor noise absorption at the high temperature of $250^{\circ} \mathrm{C}$.

\section{Supplementary Files}

This is a list of supplementary files associated with this preprint. Click to download.

- SupplementaryMovie1.avi

- SupplementaryMovie2.avi

- SupplementaryMovie3.avi

- SupportingInformation.docx

- TableofContentsGraphic.pdf 